DR. KELLY ALLOTT (Orcid ID : 0000-0002-0640-4634)

DR. TAMSYN VAN RHEENEN (Orcid ID : 0000-0003-3339-6665)

Article type : Commentary

\title{
The complexities of understanding cognitive trajectory in bipolar disorder
}

Kelly Allott ${ }^{1,2}$ and Tamsyn E Van Rheenen ${ }^{3,4}$

${ }^{1}$ Orygen, Parkville, Australia

${ }^{2}$ Centre for Youth Mental Health, University of Melbourne, Parkville, Australia

${ }^{3}$ Melbourne Neuropsychiatry Centre, Department of Psychiatry, University of Melbourne and Melbourne Health, Parkville, Australia

${ }^{4}$ Faculty of Health, Arts and Design, School of Health Sciences, Centre for Mental Health, Swinburne University, Hawthorn, Australia

The recent systematic review and meta-analysis by Szmulewicz, Valerio (1) addresses an issue of current debate in the field - that of whether cognition in bipolar disorder (BD) is neuroprogressive or stable(2). Based on a synthesis of studies of longitudinal cognitive performance in recent-onset (8 studies) and late-life BD (4 studies), this review provides evidence against this hypothesis and is consistent with an earlier meta-analysis in predominantly mid-life adults with established $\mathrm{BD}(3)$.

The extant longitudinal evidence suggests that the cognitive path of individuals with BD may be set early on. However, it is increasingly recognised that cognitive heterogeneity is the norm in BD, with cluster analytic cross-sectional studies suggesting that there are subgroups with different cognitive profiles at various stages of illness(2). There is some evidence that these profiles differ based on degree of impairment, rather than the specific cognitive domains affected. Generally, longitudinal studies have investigated cognitive trajectory This is the author manuscript accepted for publication and has undergone full peer review but has not been through the copyediting, typesetting, pagination and proofreading process, which may lead to differences between this version and the Version of Record. Please cite this article as doi: $10.1111 /$ BDI.12907

This article is protected by copyright. All rights reserved 
within $\mathrm{BD}$ as one group, but given cross-sectional cognitive heterogeneity, it is possible that latent subgroups with different cognitive trajectories exist(2). Such trajectories may be associated with different illness characteristics. For example, a recent 5-year longitudinal study found no evidence of progression of cognitive impairment in a BD cohort as a whole, but that a higher number of (hypo)manic episodes was associated with a decrease in global cognition, as well as working memory and visual memory(4). These findings tentatively suggest that individual variation in manic episodes history is relevant to variation in cognitive trajectory, which lends some longitudinal support to the neuroprogressive hypothesis of cognitive impairment in a subset of BD individuals. Teasing apart the extent to which episodic relapse actually confers cognitive decline in these patients, as opposed to the two being associated but independent features, remains a complex challenge.

Another important consideration in the context of the findings of Szmulewicz, Valerio (1), and one that has been previously acknowledged, is that longer follow-up periods may yield trajectories that do not suggest stability(2). Follow-up periods of cognitive trajectory in BD have generally been 6 years or less. To our knowledge, the longest follow-up of cognitive functioning in BD was 9 years. In this study, executive functioning (cognitive flexibility) worsened over the follow-up period; although there was no control group for comparison with age-related performance over time(2). Lessons from the schizophrenia field, which is further advanced than that of BD with respect to cognitive functioning, suggest that length of follow-up, including assessment of cognition prior to illness onset provides a different picture to studies with shorter follow-up periods. Longitudinal birth cohort studies also show that trajectory may vary according to cognitive domain, with recent evidence suggesting decline in only certain cognitive domains from premorbid (childhood) to chronic phases (adulthood) of schizophrenia(5).

Medication effects may also mediate trajectory. While this can be difficult to measure accurately over time, particularly if compliance is inconsistent, understanding the role of medication in relation to cognitive functioning should remain a goal of future studies. Medications including lithium, may either serve as neuroprotective, buffering an individual from cognitive decline, or impairing, such as antipsychotics or other medications associated with high anticholinergic load. Consideration of medication-related effects will undoubtedly help to disentangle the extent to which cognitive impairment is secondary to the outward manifestation of the illness. Exploration of cognitive, treatment and illness heterogeneity is 
also key if future studies are to uncover whether there exists a subgroup of individuals with $\mathrm{BD}$ who do experience cognitive decline and what the risk factors for decline are.

\section{References}

1. Szmulewicz A, Valerio MP, Martino DJ. Longitudinal analysis of cognitive performances in recent-onset and late-life Bipolar Disorder: A systematic review and meta-analysis. Bipolar Disorders. 2019.

2. Van Rheenen TE, Lewandowski KE, Bauer IE, Kapczinski F, Miskowiak K, Burdick KE, et al. Current understandings of the trajectory and emerging correlates of cognitive impairment in bipolar disorder: An overview of evidence. Bipolar Disorders. 2019.

3. Bora E, Ozerdem A. Meta-analysis of longitudinal studies of cognition in bipolar disorder: comparison with healthy controls and schizophrenia. Psychological Medicine. 2017; 47:2753-66. 4. Sanchez-Morla EM, Lopez-Villarreal A, Jimenez-Lopez E, Aparicio AI, Martinez-Vizcaino V, Roberto RJ, et al. Impact of number of episodes on neurocognitive trajectory in bipolar disorder patients: a 5-year follow-up study. Psychological Medicine. 2019; 49:1299-307.

5. Mollon J, David AS, Zammit S, Lewis G, Reichenberg A. Course of cognitive development from infancy to early adulthood in the psychosis spectrum. JAMA Psychiatry. 2018; 75:270-9. 


\section{University Library}

\section{- M M I E R R A A gateway to Melbourne's research publications}

Minerva Access is the Institutional Repository of The University of Melbourne

Author/s:

Allott, K;Van Rheenen, TE

Title:

The complexities of understanding cognitive trajectory in bipolar disorder

Date:

2020-04-20

Citation:

Allott, K. \& Van Rheenen, T. E. (2020). The complexities of understanding cognitive trajectory in bipolar disorder. BIPOLAR DISORDERS, 22 (5), pp.534-535. https:// doi.org/10.1111/bdi.12907.

Persistent Link:

http://hdl.handle.net/11343/275664 